\title{
Oral Appliance and Pharmacologic Agents in the Treatment of Sleep Apnea: A Pilot Clinical Study
}

\author{
Rutger Wolfgang Stache, DMD, MS ${ }^{1}$; Bharati Prasad, MD, MS²; Grace Viana, BSc, MSc ${ }^{3}$; David Carley, PhD4; Maria Therese \\ Galang-Boquiren, DMD, MS 5 \\ ${ }^{1}$ University of Illinois at Chicago, Department of Orthodontics (former resident); Private Practice, Chicago, IL; ${ }^{2}$ Associate Professor of \\ Medicine, University of Illinois at Chicago, Department of Medicine; ${ }^{3}$ Clinical Assistant Professor, University of Illinois at Chicago, \\ Department of Orthodontics; ${ }^{4}$ Professor Emeritus, University of Illinois at Chicago, Department of Biobehavioral Nursing Science; 5 \\ Associate Professor of Orthodontics, University of Illinois at Chicago, Department of Orthodontics
}

\begin{abstract}
Hypothesis: Combination therapy using a mandibular advancement device (MAD) and pharmacotherapy (ondansetron + fluoxetine) will improve therapeutic efficacy in patients with moderate to severe obstructive sleep apnea (OSA).

Methods: Twenty-one patients met inclusion criteria and were enrolled. Those with moderate-severe OSA were treated with a TAP3 Elite ${ }^{\circledR}$ MAD plus placebo medication for 2 weeks, followed by a combination regimen of fluoxetine (10 mg/day) and ondansetron ( 24 $\mathrm{mg} /$ day) with continued use of the MAD for 4 weeks.

Results: Ten patients ( 7 male and 3 female, body mass index $38.54 \pm 8.4 \mathrm{~kg} / \mathrm{m}^{2}$ ) completed the study. Apnea-hypopnea index (AHI, events per hour) MAD + pharmacotherapy (18.02 \pm 13.52$)$ was lower than the AHI baseline (32.96 \pm 10.08$)$. Mean AHI showed a statistically significant $(P=0.011)$ decrease with the combination treatment of MAD + pharmacotherapy compared to MAD + placebo. The mean oxygen desaturation index (ODI) showed a decrease from baseline (34.38 \pm 12.6$)$ compared to MAD + placebo (26.42 \pm $18.56)$ and MAD + pharmacotherapy $(18.46 \pm 15.17)$ The decrease in ODI with MAD + pharmacotherapy relative to ODI - MAD + placebo was statistically significant, $P=0.022$. The mean respiratory effort-related arousal (RERA) from baseline (7.87 \pm 5.91$)$ was higher than mean RERA index of MAD + placebo (3.45 \pm 4.00$)$ and MAD + pharmacotherapy $(2.63 \pm 2.26)$; however, the decrease in RERA index in MAD + pharmacotherapy relative to RERA at MAD + placebo was not statistically significant.
\end{abstract}

Conclusion: Combination of pharmacotherapy and oral appliance may be a viable option in treating patients with moderate to severe OSA.

Keywords: oral appliance therapy, pharmacotherapy,combination therapy,obstructive sleep apnea

Citation: Stache RW, Prasad B, Viana G, Carley D Galang-Boquiren MT. Oral appliance and pharmacologic agents in the treatment of sleep apnea: A pilot clinical study. J Dent Sleep Med. 2022;9(1)

\section{INTRODUCTION AND LITERATURE REVIEW}

Obstructive sleep apnea (OSA) is a respiratory disorder on the larger spectrum of sleep-disordered breathing (SDB). According to the American Academy of Sleep Medicine, SDB is an encompassing term for many different sleep-related breathing disorders. ${ }^{1}$ It is recognized as a major public health issue. Recent prevalence estimates of moderate to severe OSA in the United States is $14.5 \%$ among adults aged 30 to 69 years. $^{2}$

OSA is primarily characterized by repetitive and transient disruptions of breathing during sleep. These come in two forms: complete cessation and collapse of the upper airway (apnea) and a significant reduction in breathing (hypopnea). Sleep studies quantify these events into an apnea-hypopnea index (AHI), which serves as a key objective outcome measure. ${ }^{3}$ AHI scores of 5,15 , and 30 events per hour correspond with the threshold for mild, moderate, and severe sleep apnea, respectively. Extensive sleep data and physiologic measurements are routinely obtained through a polysomnogram where the patient is connected to multiple electrodes and monitored during a night of sleep. ${ }^{1,3}$

The clinical presentation of OSA includes snoring, daytime sleepiness, which can lead to impaired concentration and mood, increased risk of automobile accidents, job absenteeism, and deterioration of personal relationships. $^{4}$ Direct physiologic effects include intermittent decreases in blood oxygen saturation, which result in sympathetic nervous system activation and a subsequent increase in blood pressure, markers of oxidative stress and inflammation. ${ }^{4}$

Major risk factors for OSA are obesity and metabolic syndrome. Numerous studies have shown an independent association between OSA and hypertension, cardiovascular disease, and metabolic disease..$^{5,6}$

Continuous positive airway pressure (CPAP) devices remain the gold standard treatment for OSA because of their proven efficacy and near $100 \%$ success when properly fitted and adhered to in numerous studies over the past 
several decades. ${ }^{7}$ Despite the benefits, adherence to CPAP therapy is poor, with failure rates ranging from $29 \%$ to $83 \%$ and often greater than $50 \%$. Nonadherence is typically defined as less than 4 hours of usage per night. ${ }^{8}$ Alternative treatments include orthognathic surgery and upper airway stimulation. ${ }^{9}, 10$

Oral appliances have become a popular and more acceptable alternative to CPAP and surgical intervention. They function by repositioning the lower jaw anteriorly, which displaces oral and pharyngeal soft tissue, including the tongue, anteriorly thus increasing upper airway volume and reducing pharyngeal collapsibility. ${ }^{11,12}$ Although oral appliances are an FDA-approved alternative therapy, they are not universally effective in all patients and have varying degrees of success depending on individual anatomic factors and severity of disease. Most patients report subjective improvements in sleep quality and a reduction of daytime sleepiness. Tooth movement and malocclusion are noted in some patients especially after 1 or more years of treatment. Changes are usually reversible and can be counteracted with a morning repositioner. ${ }^{13}$

Considering the poor adherence associated with CPAP, and limited effectiveness of the oral appliance, the search for an optimal pharmacotherapeutic agent remains an elusive goal. ${ }^{14}$ Pharmaceutical agents' mechanisms of action are varied and may act through several pathways. These include increasing respiratory drive, reducing the duration of rapid eye movement (REM) stage sleep, increasing upper airway muscle tone, and changing respiratory reflexes to reduce the collapsibility of the upper airway. ${ }^{14} \mathrm{~A}$ decline in serotonergic assistance to the upper airway neurons during sleep is a possible mechanism, which leads the upper airway to easily collapse in patients with OSA. ${ }^{15}$

Combination treatment with a serotonin type-3 receptor antagonist (ondansetron) and serotonin reuptake inhibitor (fluoxetine) was well tolerated by patients with OSA and reduced the frequency of apneas and hypopneas by an average of $40 \%$ even in patients with moderate to severe disease. ${ }^{16}$ Despite partial successes in small shortterm studies, there is not enough evidence to recommend drug therapy for OSA. ${ }^{17}$

The authors of this article proposed that the muscle activation or stretching effect of the oral appliance, in this case a mandibular advancement device (MAD) will be additively enhanced by the activation of serotonergic receptors from the drugs ondansetron and fluoxetine acting on the upper airway. This addresses an unmet treatment need for patients with moderate to severe OSA who cannot tolerate the CPAP device. It is the first study to combine a dental device and pharmacotherapy.

The authors of this article also hypothesized that MAD is not fully therapeutic in moderate to severe OSA and that augmentation of MAD by pharmacotherapy (ondansetron + fluoxetine) will increase therapeutic efficacy in these patients.

\section{MATERIALS AND METHODS}

An explorative prospective single-arm study was conducted on patients with moderate to severe OSA treated initially with MADs and placebo for 2 weeks, followed by a combination regimen of the medications ondansetron + fluoxetine in addition to ongoing MAD treatment for another 4 weeks, for a total treatment period of 42 days. This protocol was approved by the University of Illinois at Chicago Institutional Review Board (IRB\# 20110629).

The primary outcome measures were derived from the polysomnogram and included treatment-related change in AHI (number of apneas or hypopneas per hour of sleep), respiratory effort-related arousals (RERA index), oxygen desaturation index (ODI), and sleep efficiency. Secondary outcome measures included: the Epworth Sleepiness Scale (ESS, a subjective self-assessment of sleepiness), ${ }^{18}$ the Functional Outcomes of Sleep Questionnaire (FOSQ; a self-assessment instrument to evaluate the effect of sleep/sleepiness on daily functioning), ${ }^{19}$ the Psychomotor Vigilance Test (PVT) to assess daytime alertness, ${ }^{20}$ and the Treatment Satisfaction Questionnaire for Medication to measure treatment acceptability. ${ }^{21}$

\section{Study Patients}

Patients were recruited from the University of Illinois at Chicago Sleep Science Center through screening of existing medical records with the following inclusion criteria: patients aged 18-64 years with confirmed diagnosis of OSA-defined AHI of 20 to 50 events per hour by baseline polysomnography, and have at least five posterior teeth in each quadrant for retention of the MAD. As this was a proof of concept, no body mass index (BMI) cutoff was used for inclusion.

Patients with the following risk factors were excluded from the study: very severe OSA warranting immediate care in the physician's medical judgment, or with oxygen saturation less than $75 \%$ for more than $5 \%$ of the sleep period time as confirmed by diagnostic polysomnogram; a history of chronic use of alcohol, narcotics, or other psychotropic drugs; treatment for OSA by any type of surgery; major surgery within 6 months; a significant defect in nasal patency due to anatomic abnormalities or uncontrolled or recurrent episodes of rhinitis; any clinically significant unstable or progressive medical condition; any primary sleep disorder other than OSA; presence or history of clinically significant chronic obstructive pulmonary disease; cardiovascular disease; gastrointestinal, respiratory, pancreatic, hepatic, renal, hematologic, endocrine (including insulin-dependent diabetes mellitus), neurologic, urogenital, connective tissue, dermatologic, thyroid, or other medical disorder; presence or history of any clinically significant psychiatric disorder; pregnancy demonstrated by positive urine test for human chorionic 
gonadotropin; current report or laboratory evidence of drug abuse; current orthodontic treatment; active periodontal disease; active temporomandibular joint disease; and evening, night, or rotating shift work.

\section{Figure 1. Flowchart of study procedures}

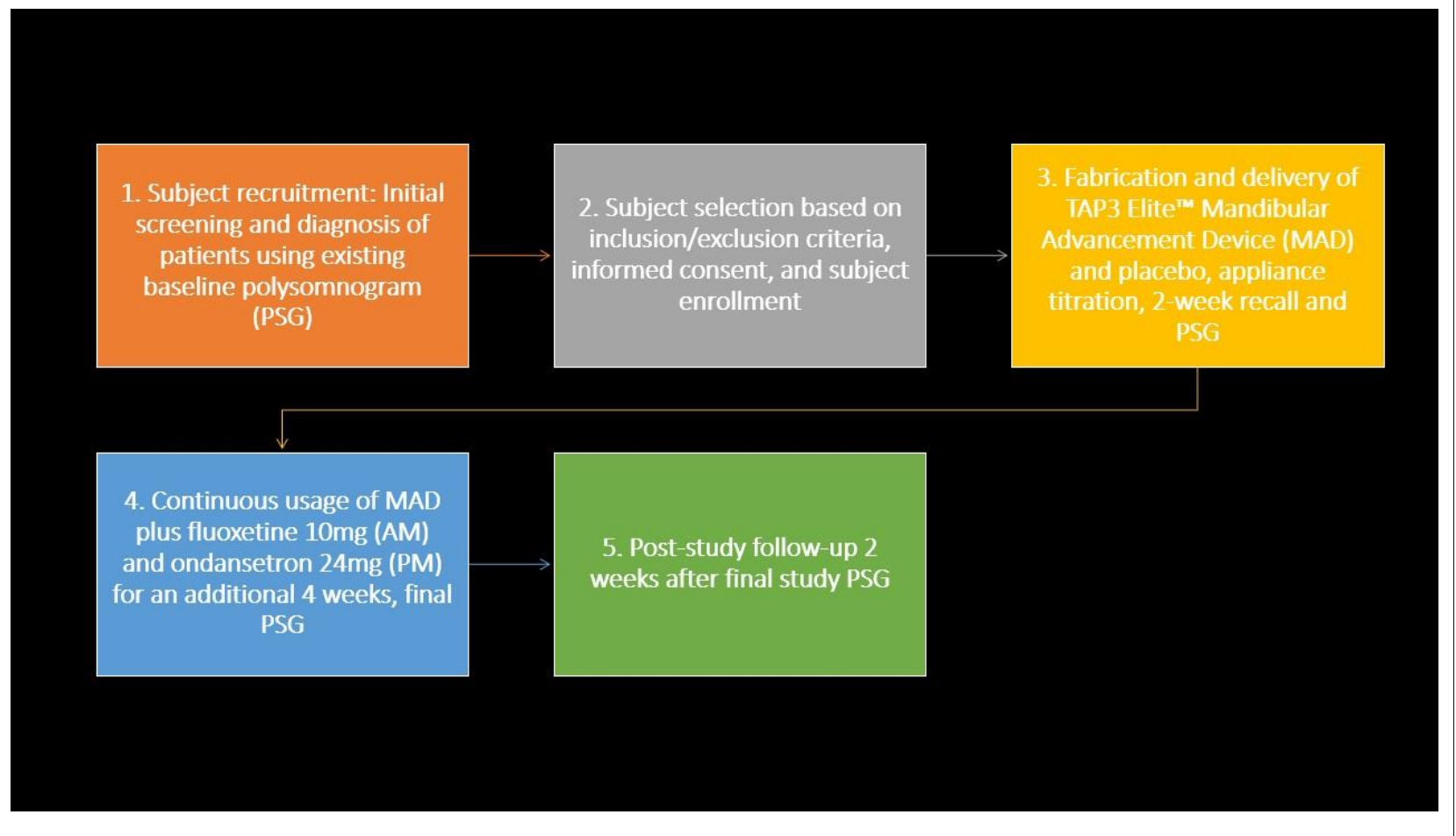

\section{Study Protocol}

Baseline sleep study records and past medical history of all patients from the University of Illinois at Chicago Sleep Science Center were examined for an AHI of 20 to 50 events per hour over a nearly 3 -year recruitment period, 2016-2019. Fifteen hundred patient medical records were filtered based on AHI range and past medical history was reviewed for initial eligibility. From those records, 228 patients were initially recruited by mail and phone call to determine study eligibility based on inclusion and exclusion criteria. Twenty-one subjects met inclusion and exclusion criteria and were enrolled in the study. Of the 21 enrolled patients, 8 were dropped from the study because they did not complete all required study visits. Two patients were dropped from the study because they were nonresponsive to oral appliance therapy per medical judgement of the consultant sleep physician after the first study polysomnogram (MAD + placebo). One patient could not tolerate the oral appliance and was excluded.

Each eligible, enrolled patient progressed through the following series of procedures (Figure 1), beginning with the initial screening visit. At the initial screening visit, the following tests were performed for determination of study eligibility: routine physical examination, medical history to determine any previous treatments that may exclude a patient from the study, urine test for drug screening and pregnancy test (if appropriate), dental examination. A baseline polysomnogram was required to determine eligibility if there has not been any within the past 2 months.

The MAD used in this study was the Thornton Adjustable Positioner - TAP3 ${ }^{\mathrm{TM}}$ Elite (Airway Management, Inc. Dallas, TX), a custom- suited, fully adjustable two-piece device. Dental assessment was performed at appliance delivery to titrate the appliance to $50 \%$ of the maximum protrusion. Patients were acclimated to this protrusion for 1 week, after which we increased protrusion to $75 \%$ of the maximum depending on patient tolerance.

The patients were also given the placebo (in identical blister packages as the actual medications) on the day of appliance delivery. After 2 weeks, the patients came back for overnight polysomnography, their empty blister packages were collected, and the patients were given a subsequent set of packages containing the actual medications. They were advised to follow the same instructions as with the placebo, that is, wearing the appliance each night before going to bed, and taking the medications in the morning with breakfast (fluoxetine $10 \mathrm{mg}$ ) and evening (ondansetron $24 \mathrm{mg}$ ) 30 minutes before going to bed.

Another overnight polysomnogram was conducted after 4 weeks of taking the medications, and finally after 7 
to 10 days, a final study visit was performed in order to collect the empty medication blister packs, conduct a final patient assessment, and collect the rest of the study data.

Table 1. Descriptive Statistics

\begin{tabular}{|c|c|c|c|c|}
\hline & Sex & $\mathrm{N}$ & Mean & Std. Error Mean \\
\hline \multirow[t]{2}{*}{ BMI $\left(\mathrm{kg} / \mathrm{m}^{2}\right)$} & Male & 7 & 34.21 & 3.02 \\
\hline & Female & 3 & 48.63 & 5.75 \\
\hline \multirow[t]{3}{*}{ Age (years) } & Male & 7 & 49.29 & 3.86 \\
\hline & Female & 3 & 47.67 & 7.36 \\
\hline & & $\mathrm{N}$ & Mean BMI & Std. Error Mean \\
\hline \multirow{2}{*}{$\begin{array}{l}\text { Positive Responder to } \\
\text { Treatment V1-7 }\end{array}$} & Yes & 8 & 36.18 & 3.77 \\
\hline & No & 2 & 48.00 & 0.00 \\
\hline
\end{tabular}

$\mathrm{BMI}=$ body mass index; Std Error Mean = standard error of the mean.

\section{Table 2. Descriptive Statistics of the Outcome Variables - Mean and Standard Deviation}

\begin{tabular}{|l|r|r|}
\hline \multicolumn{1}{|c|}{ Variables } & \multicolumn{1}{c|}{ Mean } & $\begin{array}{l}\text { Standard } \\
\text { Deviation }\end{array}$ \\
\hline AHI-baseline & 32.96 & 10.09 \\
\hline AHI-MAD + placebo & 28.38 & 17.83 \\
\hline AHI-MAD + drugs & 18.02 & 13.53 \\
\hline ODI-baseline & 34.38 & 12.62 \\
\hline ODI-MAD + placebo & 26.42 & 18.56 \\
\hline ODI-MAD + drugs & 18.46 & 15.17 \\
\hline RERA-baseline & 7.87 & 5.91 \\
\hline RERA-MAD + placebo & 3.45 & 4.00 \\
\hline RERA-MAD + drugs & 2.63 & 2.26 \\
\hline Sleep Efficiency-baseline & 65.24 & 17.12 \\
\hline Sleep Efficiency-MADD + placebo & 78.33 & 10.04 \\
\hline Sleep Efficiency- MAD+ drugs & 76.99 & 11.35 \\
\hline ESS-baseline & 10.30 & 4.60 \\
\hline ESS-MAD + placebo & 7.20 & 4.94 \\
\hline ESS-MAD + drugs & 6.30 & 5.06 \\
\hline
\end{tabular}

\section{Outcome Variables}

The following variables were recorded by overnight polysomnography at baseline (diagnosis), after MADplacebo treatment, and at the end of the study after combination drug-MAD treatment: AHI, RERA, sleep efficiency, and ODI. Sleep stages and respiratory events (apneas, hypopneas) were scored according to American Academy of Sleep Medicine criteria.

Daytime sleepiness, functional status, and daytime vigilance were assessed using the ESS, FOSQ, visual analog scale for sleepiness, and PVT, respectively. Medication satisfaction was assessed via the Treatment Satisfaction Questionnaire for Medications at specified time points.

\section{Statistical Analysis}

Test of normality by Shapiro-Wilks indicated that the study variables are normally distributed. Paired Student $t$ tests were used for mean comparisons. Statistical significance was set at 0.05 . IBM SPSS version 22.0 (IBM, Armonk, NY) was used to analyze the data. 


\section{RESULTS}

A sample of 10 patients ( 7 male and 3 female) was included in the final analysis of this study. All patients had obesity (mean BMI $38.54 \pm 8.4 \mathrm{~kg} / \mathrm{m}^{2}$ ) at baseline.

Eight of 10 patients (80\%) showed a decrease in AHI of $40 \%$ or greater from baseline compared to intervention with MAD + pharmacotherapy. These patients were defined as "positive responders" to treatment intervention. This cutoff was chosen based on the original trial of the medications, which described a $40 \%$ reduction in AHI in the high-dose ondansetron + fluoxetine group compared to baseline. ${ }^{16}$ Of those eight positive responders, six were male and two were female (Table 1). Positive responders had a lower mean BMI $\left(36.2 \pm 10.65 \mathrm{~kg} / \mathrm{m}^{2}\right)$ than nonresponders $\left(48.00 \mathrm{~kg} / \mathrm{m}^{2} \pm 0.0\right)$ (Table 1$)$.

Table 2 shows the mean AHI had a favorable decrease with the combination treatment of MAD + pharmacotherapy compared to MAD + placebo. The decrease in AHI-placebo relative to baseline was not statistically significant. However, the decrease in AHI with $\mathrm{MAD}+$ pharmacotherapy relative to $\mathrm{AHI}+$ placebo was statistically significant, $P=0.011$ (Table 3 ).

The mean ODI showed a decrease from baseline $(34.38 \pm 12.6)$ compared to MAD + placebo $(26.42 \pm$ 18.56) and MAD + pharmacotherapy (18.46 \pm 15.17$)$ (Table 2). The decrease in ODI with MAD + placebo relative to baseline was not statistically significant. However, the decrease in ODI - MAD + pharmacotherapy relative to ODI - MAD + placebo was statistically significant, $P=0.022$. (Table 3 ).

The mean RERAs from baseline $(7.87 \pm 5.91)$ was higher than mean RERA index of MAD + placebo $(3.45 \pm$ 4.00) and MAD + pharmacotherapy $(2.63 \pm 2.26)$. The mean RERA index decreased from baseline to both treatments (Table 2). However, the decrease in RERA index -MAD + pharmacotherapy relative to RERA- MAD + placebo was not statistically significant. (Table 3 ).

The mean sleep efficiency (\%) at baseline was lower than sleep efficiency $(\%)$ at MAD + placebo. This indicated that sleep efficiency improved with MAD + placebo (78.33 $\pm 10.04)$ relative to baseline $(65.24 \pm 17.12)$. The increase in sleep efficiency (\%)-MAD + placebo relative to baseline was statistically significant, $P=0.012$, although a decrease in sleep efficiency in MAD + pharmacotherapy (76.99 \pm 11.35) relative to placebo did not indicate statistical significance mean difference (Table 3 ).

The mean ESS score at baseline was higher than mean $\mathrm{ESS}$ at MAD + placebo and the MAD + pharmacotherapy (Table 2). The decrease in ESS score at MAD + placebo relative to baseline indicated a statistically significant mean difference, $P=0.047$.

PVT, mean response time, FOSQ, and visual analog scale score of sleepiness tests did not show statistical significance comparing baseline with MAD + placebo and $\mathrm{MAD}+$ placebo with MAD + pharmacotherapy.
Patient compliance with both MAD and medications were recorded via self-report and showed a mean MAD compliance rate of $6.45 \pm 1.49$ hours of wear per night for at least 6 nights per week. The medication compliance with placebo was $100 \pm 0.01 \%$ whereas the compliance with pharmacotherapy was $99 \pm .03 \%$.

\section{DISCUSSION}

The goal of this pilot study was to determine whether combination treatment of oral appliance therapy with pharmacologic intervention was a viable treatment option in patients with moderate to severe OSA. Oral appliance therapy has not been shown to be fully effective in moderate to severe OSA. In an investigation of objective success of MAD treatment, Vanderveken and colleagues described that in a population with mild to moderate OSA, oral appliance therapy successfully manages the disease in one-third of patients, lowers the AHI by half in another third of the patients, and does not produce any significant change in the remaining third. ${ }^{22}$ This study was unique because it explored the feasibility of treating a more severe OSA patient population (moderate to severe) with an oral appliance augmented with previously investigated drugs to provide a viable alternative to CPAP.

This study was quite labor intensive as the study authors designed a robust clinical study following a very stringent inclusion-exclusion criteria as outlined previously. It took almost 3 years to complete this study due to difficulty finding specific patients who met the moderate to severe AHI range of 25 to 50 years and ensuring that those patients were good candidates for oral appliance therapy. A number of patients were not eligible because of their oral health status. Once a prospective patient was consented, it was very challenging to match schedules as the study authors had to strictly schedule the 2 -week and 4-week overnight polysomnogram studies. For some patients, sudden changes in their personal schedules made these study visits inconvenient, but these were immutable time points as effects were captured after placebo and after combination therapy, respectively. To the authors' knowledge, this is the first study to investigate the combination of oral appliance therapy for OSA, particularly a MAD, with pharmacotherapy, specifically a fluoxetine - ondansetron regimen.

The results of this study are quite promising as in this small sample size, MAD + placebo showed no statistically significant difference from baseline upon examination of objective outcome variables AHI, ODI, and RERAs, but once the medication combination was added to the treatment regimen, significant differences in AHI and ODI appeared.

Night-to-night variability in polysomnogram studies is a well-established phenomenon. ${ }^{23}$ Stöberl and colleagues followed 77 patients with OSA for 2 weeks with pulse 
oximetry and found significant variability in severity as measured by AHI from night to night. ${ }^{24}$ This could explain the increase in AHI for 5 of the 10 patients from baseline to $\mathrm{MAD}+$ placebo. But more importantly, when comparing the AHI scores at MAD + placebo to those at MAD + pharmacotherapy, 8 of 10 patients showed a decrease in AHI. This suggests that the combination intervention had a positive effect on reducing the number

\section{Table 3. Paired Mean Differences}

\begin{tabular}{|c|c|c|c|c|c|}
\hline Variables & Mean & $\begin{array}{l}\text { Standard } \\
\text { Deviation }\end{array}$ & $\begin{array}{l}\text { Mean } \\
\text { Difference }\end{array}$ & $\begin{array}{l}\text { 95\% Confidence } \\
\text { Interval }\end{array}$ & $P$ \\
\hline AHI-baseline & 32.96 & 10.09 & \multirow[b]{2}{*}{4.58} & \multirow[b]{2}{*}{$(-10.30,19.45)$} & \multirow[b]{2}{*}{0.504} \\
\hline AHI-MAD + placebo & 28.38 & 17.83 & & & \\
\hline AHI-MAD + placebo & 28.38 & 17.83 & \multirow[b]{2}{*}{10.36} & \multirow[b]{2}{*}{$(3.01,17.71)$} & \multirow[b]{2}{*}{$0.011^{\mathrm{a}}$} \\
\hline AHI-MAD + drugs & 18.02 & 13.53 & & & \\
\hline ODI-baseline & 34.38 & 12.62 & \multirow[b]{2}{*}{7.96} & \multirow[b]{2}{*}{$(-9.55,25.47)$} & \multirow[b]{2}{*}{0.331} \\
\hline ODI-MAD + placebo & 26.42 & 18.56 & & & \\
\hline ODI-MAD + placebo & 26.42 & 18.56 & \multirow[b]{2}{*}{7.96} & \multirow[b]{2}{*}{$(1.48,14.44)$} & \multirow[b]{2}{*}{$0.022^{\mathrm{a}}$} \\
\hline ODI-MAD + drugs & 18.46 & 15.17 & & & \\
\hline RERA-baseline & 7.87 & 5.91 & \multirow[b]{2}{*}{4.42} & \multirow[b]{2}{*}{$(-1.19,10.03)$} & \multirow[b]{2}{*}{0.109} \\
\hline RERA-MAD + placebo & 3.45 & 4.00 & & & \\
\hline RERA-MAD + placebo & 3.45 & 4.00 & \multirow[b]{2}{*}{0.82} & \multirow[b]{2}{*}{$(-2.22,3.86)$} & \multirow[b]{2}{*}{0.557} \\
\hline RERA-MAD + drugs & 2.63 & 2.26 & & & \\
\hline Sleep efficiency-baseline & 65.24 & 17.12 & \multirow[b]{2}{*}{-13.09} & \multirow[b]{2}{*}{$(-22.49,-3.69)$} & \multirow[b]{2}{*}{$0.012^{\mathrm{a}}$} \\
\hline $\begin{array}{lll}\begin{array}{l}\text { Sleep } \\
\text { placebo }\end{array} & \text { efficiency-MAD }+ \\
\end{array}$ & 78.33 & 10.04 & & & \\
\hline $\begin{array}{l}\text { Sleep efficiency-MAD } \quad+ \\
\text { placebo }\end{array}$ & 78.33 & 10.04 & \multirow[b]{2}{*}{1.34} & \multirow[b]{2}{*}{$(-7.96,10.64)$} & \multirow[b]{2}{*}{0.752} \\
\hline Sleep efficiency-MAD + drugs & 76.99 & 1.35 & & & \\
\hline ESS-baseline & 10.30 & 4.60 & \multirow[b]{2}{*}{3.10} & \multirow[b]{2}{*}{$(0.57,6.14)$} & \multirow[b]{2}{*}{$0.047^{\mathrm{a}}$} \\
\hline ESS-MAD + placebo & 7.20 & 4.94 & & & \\
\hline ESS-MAD + placebo & 7.20 & 4.94 & \multirow[b]{2}{*}{0.90} & \multirow[b]{2}{*}{$(-0.77,2.57)$} & \multirow[b]{2}{*}{0.253} \\
\hline ESS-MAD + drugs & 6.30 & 5.06 & & & \\
\hline
\end{tabular}

a Statistically significant at $5 \%$.

$\mathrm{AHI}=$ apnea-hypopnea index; ESS = Epworth Sleepiness Scale; MAD = mandibular advancement device; ODI = oxygen desaturation index; RERA = respiratory effect-related arousal.

of apneas and hypopneas experienced during sleep, assuming a stationary effect of MAD by the time the pharmacotherapy was introduced.

Eight patients had a decrease in AHI of $40 \%$ or greater from baseline to the end of the study. They were defined as positive responders. No report of adverse drug side effects was recorded from patient study logs, and both oral appliances and medications were well tolerated, and with high compliance rates. Some patients reported mild jaw soreness the morning after wearing the oral appliance, but this subsided after 1 to 2 weeks of wear. Recent literature suggests that side effects of oral appliances, though fairly common, are mostly manageable by a qualified dentist. ${ }^{13}$

Response to treatment varied significantly with all patients. For oral appliances, the best responders tend to have mild to moderate OSA, have a greater amount of 
advancement in their appliance, have a lower BMI, and have a greater difference in respiratory events between supine and lateral sleep positions. ${ }^{11}$ In this pilot study, some of these aforementioned factors were not met by the chosen study population; the patients had moderate to severe OSA, and had higher BMI. It is possible that the therapeutic actions of the medications helped balance the inadequate effects of oral appliance therapy in this patient cohort.

The initial trial for fluoxetine-ondansetron combination followed a 28 -day study protocol. ${ }^{16}$ The onset of action of ondansetron is almost immediate, peaking at around 2 hours postingestion, whereas it takes much longer for fluoxetine. It is widely accepted that the full therapeutic benefit of fluoxetine is not reached until approximately 4 to 6 weeks. For this reason, the patients remained on the combination drug treatment for 28 days, similar to the medication combination trial adopted by Prasad et al., before undergoing the last polysomnogram of the study. ${ }^{16}$

It is important to note that AHI is not the only important variable recorded during sleep. There are also inherent problems with the AHI. It is an imperfect outcome measure. There is no quantification of work of breathing. There is also no differentiation between short and long events. AHI may or may not be the optimal metric to evaluate sleep disordered breathing. Additionally, some patients may experience a greater proportion of apneas and hypopneas during specific stages (REM-related OSA) or positions of sleep such as during supine sleep. ${ }^{25}$ Most patients showed improvement in AHI from MAD + placebo to $\mathrm{MAD}+$ pharmacotherapy, and from baseline to MAD + pharmacotherapy. Severity was reduced from severe to moderate in 6 patients and mild in 1 patient. Because treatment was not fully effective, patients were referred to their sleep physician for follow-up evaluation and treatment.

RERAs can be thought of as a milder form of an apnea or hypopnea. It defined as a subtle fluctuation in airway of $1 \%$ to $2 \%$, lasting 10 seconds or longer, and leads to an arousal or decrease in oxygen saturation. ${ }^{26}$ The significance of this is that these are not captured in the AHI score. They are an important outcome measure that can affect overall sleep quality and restfulness. The data showed a statistically significant decrease in RERA index from baseline, despite not showing the same with AHI. This highlights the fact that AHI may not be the only important outcome measure to assess when evaluating treatment efficacy of an intervention.

Sleep efficiency is a measure of time spent sleeping compared to the time spent in bed. ${ }^{27}$ There was a statistically significant increase in sleep efficiency with the $\mathrm{MAD}+$ placebo compared to baseline; however, no further difference was found upon introduction of pharmacotherapy. This finding is similar to what Pitarch and colleagues found in their clinical trial with the use of MAD compared to baseline. ${ }^{28}$
ODI is another important sleep variable, which measures the number of drops in blood oxygen levels throughout the night. ${ }^{29}$ This may or may not be correlated with the number of sleep arousals and can be predictive of long-term cardiovascular risks such as hypertension, stroke, and heart attack. ${ }^{29}$ A statistically significant decrease in ODI was found between MAD + placebo and $\mathrm{MAD}+$ medications.

The patient pool had a very high initial average BMI (38.54 \pm 3.4 ), which is reflective of the OSA population at the authors' institution. MADs have been shown to decrease in effectiveness as BMI and neck circumference increases. ${ }^{30}$ One patient with a BMI of $61 \mathrm{~kg} / \mathrm{m}^{2}$ showed a poor response to treatment and was discontinued in this study.

Subjective outcome measures included the ESS, FOSQ, visual analog scale, and Treatment Satisfaction Questionnaire for Medication. All outcome measures showed some improvement from baseline to the end of the study, although statistical significance was found only with the ESS, which is a well-validated outcome measure. The study patients exhibited a mean ESS score less than 11 at baseline; however, an important point to note is the clinical significance of the decrease in ESS scores at subsequent time points. Any decrease greater than two to three points in the ESS scores lead to clinically significant changes in daytime sleepiness. ${ }^{31}$ Thus, there was a clinically significant change in daytime sleepiness with MAD + placebo compared to baseline. It remains to be elucidated if prolonged use of the medications would have allowed such a significant change with MAD + medications.

Interestingly, all of the study patients reported feeling improvements in daytime sleepiness and increased energy level with the combination intervention of MAD + pharmacotherapy. This is contradictory to some of the observed sleep data. These sleep data were recorded at two time points during the study 4 weeks apart, and only provides short-term picture of the response to treatment. A much longer follow-up period would be ideal to determine how patients adapt to the treatment modality. How much of this effect is due to pharmacotherapy alone also remains to be determined.

Data were collected on robust variables and the feasibility of the intervention was successfully established. However, the scope and funding of the pilot study were limited. It was difficult to find suitable patients who fit all inclusion and exclusion criteria and were willing to complete the entire study. Patients were recruited from a single sleep center, which may have reduced the diversity of the patient pool.

The study results highlight the complexity of OSA and illustrate the importance of identifying traits that may predict treatment response to MAD therapy. Future studies need to be adequately powered to draw conclusions about the effectiveness of combination MAD and pharmacotherapy in order to serve the unmet need of 
finding a practical treatment alternative for the patient population with moderate to severe OSA.

\section{CONCLUSIONS}

This study determined that augmentation of oral appliances by pharmacotherapy (ondansetron + fluoxetine) may increase efficacy of oral appliance therapy. A combination of pharmacotherapy and oral appliance may be a viable option in treating patients with moderate to severe OSA. Patients generally reported sleeping better with the oral appliance and having increased alertness during the day on their follow-up appointment. Further larger scale studies based on effect sizes gathered from these data will help to provide more understandable relationships among the outcome measures of interest in this type of therapy for sleep apnea.

\section{ABBREVIATIONS}

$\begin{array}{ll}\text { AHI } & \text { Apnea-hypopnea Index } \\ \text { CPAP } & \text { Continuous positive airway pressure } \\ \text { ESS } & \text { Epworth Sleepiness Scale } \\ \text { FOSQ } & \begin{array}{l}\text { Functional Outcomes of Sleep } \\ \text { Questionnaire } \\ \text { Mandibular advancement device }\end{array} \\ \text { ODI } & \text { Oxygen desaturation index } \\ \text { OSA } & \text { Obstructive sleep apnea } \\ \text { PVT } & \text { Psychomotor Vigilance Test } \\ \text { RERA } & \text { Respiratory effort-related arousal } \\ \text { SBD } & \text { Sleep-disordered breathing } \\ & \text { ACKNOWLEDGMENTS }\end{array}$

The authors gratefully acknowledge the University of Illinois at Chicago and the American Association of Orthodontists Foundation for funding this project.

Portions of this paper were derived from a published thesis at indigo.uic.edu by Rutger W. Stache.

The authors wish to thank Drs. Naveen Ashwathanarayana, Amitoj Mehta, Ayas Makki, Joseph Deek, and Fatjona Aliaj for all their research assistance, as well as Professor Marlos Viana for reviewing the manuscript and providing statistical input. We are likewise sincerely grateful for the constructive feedback of the editors and reviewers.

\section{REFERENCES}

1. Kapur VK, Auckley DH, Chowdhuri S, et al. Clinical practice guideline for diagnostic testing for adult obstructive sleep apnea: An American academy of sleep medicine clinical practice guideline. $J$ Clin Sleep Med. 2017;13(3):479-504.

2. Benjafield AV, Ayas NT, Eastwood PR, et al. Estimation of the global prevalence and burden of obstructive sleep apnoea: a literature-based analysis. Lancet Respir Med. 2019;7(8):687-698.

3. Greenstone M, Hack M. Obstructive sleep apnoea. BMJ. 2014;348:g3745.

4. McEvoy RD, Antic NA, Heeley E, et al. CPAP for prevention of cardiovascular events in obstructive sleep apnea. $N$ Engl $\mathrm{J}$ Med. 2016;375(10):919-931.

5. Hou H, Zhao Y, Yu W, et al. Association of obstructive sleep apnea with hypertension: A systematic review and meta-analysis. J Glob Health. 2018 Jun;8(1):010405.

6. Zhang X, Fan J, Guo Y, et al. Association between obstructive sleep apnoea syndrome and the risk of cardiovascular diseases: an updated systematic review and dose-response meta-analysis. Sleep Med. $2020 \mathrm{Jul} ; 71: 39-46$

7. Carberry JC, Jordan AS, White DP, Wellman A, Eckert DJ. Upper airway collapsibility (pcrit) and pharyngeal dilator muscle activity are sleep-stage dependent. Sleep. 2016;39(3):511-521.

8. Carberry JC, Amatoury J, Eckert DJ. Personalized management approach for OSA. Chest. 2018;153(3):744-755.

9. Lorenzi-Filho G, Almeida FR, Strollo PJ. Treating OSA: Current and emerging therapies beyond CPAP. Respirology. 2017;22(8):1500-1507.

10. Noller MW, Guilleminault C, Gouveia CJ, et al. Mandibular advancement for adult obstructive sleep apnea: A systematic review and meta-analysis. J Craniomaxillofac Surg. 2017;45(12):20352040.

11. Marklund M, Braem MJA, Verbraecken J. Update on oral appliance therapy. Eur Respir Rev. 2019;28(153):190083.

12. $\mathrm{Ng} \mathrm{JH}$, Yow M. Oral appliances in the management of obstructive sleep apnea. Sleep Med Clin. 2020;15(2):241-250.

13. Sheats RD. Management of side effects of oral appliance therapy for sleep-disordered breathing: summary of American Academy of Dental Sleep Medicine recommendations. J Clin Sleep Med. 2020 May 15;16(5):835.

14. Schütz SG, Dunn A, Braley TJ, Pitt B, Shelgikar AV. New frontiers in pharmacologic obstructive sleep apnea treatment: A narrative review. Sleep Med Rev. 2021 Mar 13;57:101473.

15. Heym J, Steinfels GF, Jacobs BL. Activity of serotonin-containing neurons in the nucleus raphe pallidus of freely moving cats. Brain Res. 1982;251(2):259-276.

16. Prasad B, Radulovacki M, Olopade C, Herdegen JJ, Logan T, Carley DW. Prospective trial of efficacy and safety of ondansetron and fluoxetine in patients with obstructive sleep apnea syndrome. Sleep. 2010;33(7):982-989.

17. Mason M, Welsh EJ, Smith I. Drug therapy for obstructive sleep apnoea in adults. The Cochrane database of systematic reviews. 2013(5):CD003002.

18. Walker NA, Sunderram J, Zhang P, Lu SE, Scharf MT. Clinical utility of the Epworth sleepiness scale. Sleep Breath. 2020;24(4):1759-1765.

19. Weaver TE, Laizner AM, Evans LK, et al. An instrument to measure functional status outcomes for disorders of excessive sleepiness. Sleep. 1997;20(10):835-843.

20. Kainulainen S, Duce B, Korkalainen H, et al. Severe desaturations increase psychomotor vigilance task-based median reaction time and number of lapses in obstructive sleep apnoea patients. Eur Respir J. 2020;55(4):1901849.

21. Bharmal M, Payne K, Atkinson MJ, Desrosiers MP, Morisky DE, Gemmen E. Validation of an abbreviated Treatment Satisfaction Questionnaire for Medication (TSQM-9) among patients on antihypertensive medications. Health Qual Life Outcomes. 2009;7:36.

22. Vanderveken OM, Dieltjens M, Wouters K, De Backer WA, Van de Heyning PH, Braem MJ. Objective measurement of compliance during oral appliance therapy for sleep-disordered breathing. Thorax. 


\section{3;68(1):91-96}

23.

Prasad B, Usmani S, Steffen AD, et al. Short-term variability in apnea-hypopnea index during extended home portable monitoring. $J$ Clin Sleep Med. 2016;12(6):855-863.

24. Stöberl AS, Schwarz EI, Haile SR, et al. Night-to-night variability of obstructive sleep apnea. J Sleep Res. 2017;26(6):782-788.

25. Duce B, Kulkas A, Langton C, Töyräs J, Hukins C. The prevalence of REM-related obstructive sleep apnoea is reduced by the AASM 2012 hypopnoea criteria. Sleep Breath. 2018;22(1):57-64.

26. Pépin JL, Guillot M, Tamisier R, Lévy P. The upper airway resistance syndrome. Respiration. 2012;83(6):559-566.

27. Reed DL, Sacco WP. Measuring sleep efficiency: What should the denominator be?. J Clin Sleep Med. 2016;12(2):263-266.

28. Pitarch MR, García MS, Cuesta JP, Algarra JM, Fernández JE, Fons Font A. Effectiveness of a mandibular advancement device in obstructive sleep apnea patients: a prospective clinical trial. Eur Arch Otorhinolaryngol. 2018;275(7):1903-1911.

29. Temirbekov D, Güneş S, Yazıcı ZM, Sayın İ. The ignored parameter in the diagnosis of obstructive sleep apnea syndrome: The oxygen desaturation index. Turk Arch Otorhinolaryngol. 2018;56(1):1.

30. Sutherland K, Chan ASL, Cistulli PA. Three-dimensional assessment of anatomical balance and oral appliance treatment outcome in obstructive sleep apnoea. Sleep Breath. 2016;20(3):903910
31. Patel S, Kon SSC, Nolan CM, et al. The Epworth Sleepiness Scale: Minimum clinically important difference in obstructive sleep apnea. Am J Respir Crit Care Med. 2018;197(7):961-963.

\section{SUBMISSION \& CORRESPONDENCE INFORMATION}

\section{Submitted January 15, 2021 \\ Submitted in final revised form July 07, 2021 \\ Accepted for publication September 17, 2021}

Address correspondence to: Maria Therese GalangBoquiren, 801 S. Paulina St. Room 131 M/C 841, Chicago, IL 60612; Email: mgalang@uic.edu

\section{DISCLOSURE STATEMENT}

The authors report no conflicts of interest. 